\title{
$A_{F B}^{b}$ and $R_{b}$ at CERN LEP and new right-handed gauge bosons
}

\author{
Xiao-Gang $\mathrm{He}^{*}$ \\ Department of Physics, National Taiwan University, Taipei, Taiwan 10764, Republic of China \\ G. Valencia ${ }^{\dagger}$ \\ Department of Physics, Iowa State University, Ames, Iowa 50011, USA
}

(Received 28 April 2003; published 14 August 2003)

\begin{abstract}
We explore models with additional right-handed gauge bosons that couple predominantly to the third generation in the context of $b \bar{b}$ production at CERN LEP. In particular we investigate potential new contributions to $\delta g_{R b}$ that are needed if the measured $A_{F B}^{b}$ at the $Z$ peak is interpreted as a signal of new physics. We identify two sources of large $\delta g_{R b}$ corrections: $Z-Z^{\prime}$ mixing at the tree level, and one-loop effects from a new $S U_{R}(2)$ triplet of gauge bosons. We find that the latter can contribute to $\delta g_{R b}$ at the $1 \%$ level. We place bounds on the mass of the additional $Z^{\prime}$ gauge boson that occurs in these models using the $R_{b}$ measurements from LEP-II. We find that even in cases where the $Z^{\prime}$ couples almost exclusively to the $b$ and $t$ quarks, masses lighter than about $500 \mathrm{GeV}$ are already excluded.
\end{abstract}

DOI: 10.1103/PhysRevD.68.033011

PACS number(s): 12.15.-y, 12.60.-i, 14.70.-e

\section{INTRODUCTION}

The precision measurements at the $Z$ resonance continue to exhibit a deviation from the standard model in the observable $A_{F B}^{b}$ by about -3.2 standard deviations [1,2]. At the same time $R_{b}$ deviates from the standard model by only 1.4 standard deviations [2]. It has been pointed out by Chanowitz $[3,4]$ that the deviation in $A_{F B}^{b}$ presents a problem for the standard model whether it is genuine or not. In particular, Chanowitz argues that if the anomaly in $A_{F B}^{b}$ is attributed to systematic error and dropped from the CERN $e^{+} e^{-}$LEP fits, then the indirect determination of the Higgs boson mass is in conflict with the direct limit $[3,4]$. One possible interpretation of this result is that there is new physics associated with the $Z b \bar{b}$ couplings, and we explore this possibility in the context of non-universal right-handed interactions.

We adopt the following notation for the effective couplings between the $Z$ boson and the $b$ and $t$ quarks:

$$
\mathcal{L}=-\frac{g}{2 \cos \theta_{W}} \bar{f} \gamma^{\mu}\left[\left(g_{L f}+\delta g_{L f}\right) P_{L}+\left(g_{R f}+\delta g_{R f}\right) P_{R}\right] f Z_{\mu}
$$

with $P_{L, R}=\left(1 \mp \gamma_{5}\right) / 2$, and tree-level standard model couplings $g_{L f}$ and $g_{R f}$ as in the Appendix. In terms of these effective couplings, the results in Ref. [1] suggest that new physics could be responsible for as much as $\delta g_{R b} \sim 0.04$, $\delta g_{L b} \sim 0.004$. At the same time, new physics contributions to the $\tau$-lepton couplings are constrained to be at most at the 0.001 level.

Several discussions of new physics effects regarding $A_{F B}^{b}$ or $R_{b}$ have appeared in the literature. Among them are light supersymmetry partners [5] quark mixing with new fermions [6-9] top-color [10-12] top-flavor [13-16] and non-

\footnotetext{
*Email address: hexg@phys.ntu.edu.tw

†Email address: valencia@iastate.edu
}

universal left-right models [17]. Our goal in this paper is to extend our results in Ref. [17] by computing the dominant one-loop effects to $\delta g_{R b}$, and by using the LEP-II data on $e^{+} e^{-} \rightarrow b \bar{b}$ to constrain the mass of the new gauge bosons.

In the standard model the one-loop corrections to $\delta g_{L b}$ that are proportional to $M_{t}^{2}$ are $\approx 0.006$ [18]. We can use this result as a benchmark for $\delta g_{R b}$ from new physics, suggesting that if it is to occur at one-loop there must be an enhancement relative to the standard model electroweak corrections. This is precisely what can occur in models such as those we discussed in Ref. [17], where the coupling strength of the new right-handed interaction, $g_{R}$, is significantly larger than the $S U(2)_{L}$ coupling $g_{L}$. In this paper we calculate these corrections in a simple case and find that $\delta g_{R b}$ from one-loop effects can be $1 \%$.

Our paper is organized as follows. In Sec. II we describe models with additional right-handed gauge bosons that could change the value $\delta g_{R b}$ significantly while respecting other phenomenological constraints. In particular we discuss several ways in which the predominant effects occur for the $b$ and $t$-quark couplings but not for the $\tau$-lepton couplings through $Z-Z^{\prime}$ mixing. In Sec. III we show how, even in the absence of $Z-Z^{\prime}$ mixing, one-loop contributions to $\delta g_{R b}$ can occur at the $1 \%$ level. In Sec. IV we present bounds on the mass of the new gauge bosons from the process $e^{+} e^{-}$ $\rightarrow b \bar{b}$ at LEP-II. We state our conclusions in Sec. V and relegate some details to the Appendix.

\section{NON-UNIVERSAL LEFT-RIGHT MODELS}

The models to be discussed are variations of left-right models $[19,20]$ in which the right-handed interactions single out the third generation. Our basic model was introduced in Ref. [17] and we start by recalling its salient features. The gauge group of the model is $S U(3) \times S U(2)_{L} \times S U(2)_{R}$ $\times U(1)_{B-L}$ with gauge couplings $g_{3}, g_{L}, g_{R}$ and $g$, respectively. The model differs from other left-right models in the transformation properties of the fermions. 
The first two generations are chosen to have the same transformation properties as in the standard model with $U(1)_{Y}$ replaced by $U(1)_{B-L}$,

$$
\begin{aligned}
& Q_{L}=(3,2,1)(1 / 3), \quad U_{R}=(3,1,1)(4 / 3), \\
& D_{R}=(3,1,1)(-2 / 3), \\
& L_{L}=(1,2,1)(-1), \quad E_{R}=(1,1,1)(-2) .
\end{aligned}
$$

The numbers in the first parentheses are the $S U(3)$, $S U(2)_{L}$ and $S U(2)_{R}$ group representations, respectively, and the number in the second parentheses is the $U(1)_{B-L}$ charge.

The third generation is chosen to transform differently,

$$
\begin{array}{ll}
Q_{L}(3)=(3,2,1)(1 / 3), & Q_{R}(3)=(3,1,2)(1 / 3), \\
L_{L}(3)=(1,2,1)(-1), & L_{R}=(1,1,2)(-1) .
\end{array}
$$

The above assignments are unusual compared with the conventional left-right model, but they enhance the difference between the right-handed couplings of the first two and the third generations. This model is anomaly free.

The correct symmetry breaking and mass generation of particles can be induced by the vacuum expectation values of three Higgs boson representations: $H_{R}=(1,1,2)(-1)$, whose non-zero vacuum expectation value (VEV) $v_{R}$ breaks the group down to $S U(3) \times S U(2) \times U(1)$, and the two Higgs boson multiplets, $H_{L}=(1,2,1)(-1)$ and $\phi$ $=(1,2,2)(0)$, which break the symmetry to $S U(3)$ $\times U(1)_{e m}$. For the purpose of symmetry breaking, only one of $H_{L}$ or $\phi$ is sufficient, but both are required to give masses to all fermions. It is possible to introduce additional Higgs boson representations as mentioned in Ref. [17], but we will not do so in this paper.

The introduction of $\phi$ causes the standard model $W_{L}$ and $Z_{o}$ to mix with the new $W_{R}$ and $Z_{R}$ gauge bosons. Here $W_{R}$ is the $S U(2)_{R}$ charged gauge boson and $Z_{R}$ is a linear combination of the neutral component of the $S U(2)_{R}$ gauge boson $W_{3 R}$ and the $U(1)_{B-L}$ gauge boson $B$. Specifically,

$$
\begin{aligned}
& Z_{o}=\cos \theta_{W} W_{3 L}-\sin \theta_{W} \cos \theta_{R} B-\sin \theta_{W} \sin \theta_{R} W_{3 R}, \\
& Z_{R}=\cos \theta_{R} W_{3 R}-\sin \theta_{R} B,
\end{aligned}
$$

where $\tan \theta_{R}=g / g_{R}$.

In the bases $\left(W_{L}, W_{R}\right)$ and $\left(Z_{o}, Z_{R}\right)$ for the massive gauge bosons, the mass matrices were given in Ref. [17] and we reproduce them here for later convenience:

$$
\begin{aligned}
& m_{11 W}^{2}=\frac{1}{2} g_{L}^{2}\left(\left|v_{L}\right|^{2}+\left|v_{1}\right|^{2}+\left|v_{2}\right|^{2}\right), \quad m_{22 W}^{2}=\frac{1}{2} g_{R}^{2}\left(\left|v_{R}\right|^{2}+\left|v_{1}\right|^{2}+\left|v_{2}\right|^{2}\right), \\
& m_{12 W}^{2}=-g_{L} g_{R} \operatorname{Re}\left(v_{1} v_{2}^{*}\right), \quad m_{11 Z}^{2}=\frac{1}{2} \frac{g_{L}^{2}}{\cos ^{2} \theta_{W}}\left(\left|v_{L}\right|^{2}+\left|v_{1}\right|^{2}+\left|v_{2}\right|^{2}\right), \\
& m_{22 Z}^{2}=\frac{1}{2} \frac{g_{R}^{2}}{\cos ^{2} \theta_{R}}\left[\left|v_{L}\right|^{2} \sin ^{4} \theta_{R}+\left(\left|v_{1}\right|^{2}+\left|v_{2}\right|^{2}\right) \cos ^{4} \theta_{R}+\left|v_{R}\right|^{2}\right], \\
& m_{12 Z}^{2}=\frac{1}{4} g_{L} g_{R} \frac{\sin \theta_{R}}{\cos \theta_{W}}\left[\left|v_{L}\right|^{2} \tan \theta_{R}-\left(\left|v_{1}\right|^{2}+\left|v_{2}\right|^{2}\right) \cot \theta_{R}\right] .
\end{aligned}
$$

After diagonalization of the mass-squared matrices, the lighter and heavier mass eigenstates $\left(Z, Z^{\prime}\right)$ and $\left(W, W^{\prime}\right)$ are given by

$$
\begin{aligned}
\left(\begin{array}{l}
W \\
W^{\prime}
\end{array}\right) & =\left(\begin{array}{ll}
\cos \xi_{W} & \sin \xi_{W} \\
-\sin \xi_{W} & \cos \xi_{W}
\end{array}\right)\left(\begin{array}{l}
W_{L} \\
W_{R}
\end{array}\right), \\
\left(\begin{array}{l}
Z \\
Z^{\prime}
\end{array}\right) & =\left(\begin{array}{ll}
\cos \xi_{Z} & \sin \xi_{Z} \\
-\sin \xi_{Z} & \cos \xi_{Z}
\end{array}\right)\left(\begin{array}{l}
Z_{o} \\
Z_{R}
\end{array}\right),
\end{aligned}
$$

where $\xi_{Z, W}$ are the mixing angles,

$$
\tan \left(2 \xi_{W, Z}\right)=\frac{2 m_{12(W, Z)}^{2}}{m_{11(Z, W)}^{2}-m_{22(Z, W)}^{2}} .
$$

In this model there are new interactions between the massive gauge bosons and quarks. For the charged current inter- action, there are both left and right handed interactions. In the weak eigenstate basis, the charged gauge boson $W_{L}$ couples to all generations, but the charged gauge boson $W_{R}$ only couples to the third generation. There is a similar pattern for the neutral gauge interactions. This pattern gives rise to interactions between the fermions and the lightest physical gauge bosons that can be made to resemble the standard model couplings plus enhanced right-handed couplings for the third generation. In the mass eigenstate basis the quarkgauge-boson interactions are given by

$$
\begin{aligned}
\mathcal{L}_{W}= & -\frac{g_{L}}{\sqrt{2}} \bar{U}_{L} \gamma^{\mu} V_{K M} D_{L}\left(\cos \xi_{W} W_{\mu}^{+}-\sin \xi_{W} W_{\mu}^{\prime+}\right) \\
& -\frac{g_{R}}{\sqrt{2}} \bar{u}_{R i} \gamma^{\mu} V_{R t i}^{u *} V_{R b j}^{d} d_{R j}\left(\sin \xi_{W} W_{\mu}^{+}+\cos \xi_{W} W_{\mu}^{\prime+}\right) \\
& + \text { H.c., }
\end{aligned}
$$


where $U=(u, c, t)$ and $D=(d, s, b) . V_{K M}$ is the KobayashiMaskawa mixing matrix and $V_{R i j}^{u, d}$ are unitary matrices which rotate the right-handed quarks $u_{R i}$ and $d_{R i}$ from the weak eigenstate basis to the mass eigenstate basis. The repeated indices $i$ and $j$ are summed over three generations. For the neutral sector the couplings are

$$
\begin{aligned}
\mathcal{L}_{Z}= & -\frac{g_{L}}{2 \cos \theta_{W}} \bar{q} \gamma^{\mu}\left(g_{V}-g_{A} \gamma_{5}\right) q\left(\cos \xi_{Z} Z_{\mu}-\sin \xi_{Z} Z_{\mu}^{\prime}\right) \\
& +\frac{g_{Y}}{2} \tan \theta_{R}\left(\frac{1}{3} \bar{q}_{L} \gamma^{\mu} q_{L}+\frac{4}{3} \bar{u}_{R i} \gamma^{\mu} u_{R i}-\frac{2}{3} \bar{d}_{R i} \gamma^{\mu} d_{R i}\right) \\
& \times\left(\sin \xi_{Z} Z_{\mu}+\cos \xi_{Z} Z_{\mu}^{\prime}\right)-\frac{g_{Y}}{2}\left(\tan \theta_{R}+\cot \theta_{R}\right) \\
& \times\left(\bar{u}_{R i} \gamma^{\mu} V_{R t i}^{u *} V_{R t j}^{u} u_{R j}-\bar{d}_{R i} \gamma^{\mu} V_{R b i}^{d *} V_{R b j}^{d} d_{R j}\right) \\
& \times\left(\sin \xi_{Z} Z_{\mu}+\cos \xi_{Z} Z_{\mu}^{\prime}\right) .
\end{aligned}
$$

In this expression, $g_{Y}=g \cos \theta_{R}=g_{R} \sin \theta_{R}, q$ and $q_{L}$ are summed over $u, d, c, s, t, b$ quarks, and repeated $i, j$ indices are summed over the three generations. The first line contains the standard model couplings to the $Z$ in the limit $\xi_{Z}$ $=0$. The first two lines also contain couplings of the two $Z$ bosons to quarks that arise through mixing of the neutral gauge bosons.

Similarly, the couplings to leptons are given, in the weak eigenstate basis, by

$$
\begin{aligned}
\left.\mathcal{L}_{Z} \text { (lepton) }\right)= & -\frac{g_{L}}{2 \cos \theta_{W}} \bar{\ell} \gamma^{\mu}\left(g_{V}-g_{A} \gamma_{5}\right) \ell\left(\cos \xi_{Z} Z_{\mu}\right. \\
& \left.-\sin \xi_{Z} Z_{\mu}^{\prime}\right)+\frac{g_{Y}}{2} \tan \theta_{R}\left(-\bar{\ell}_{L} \gamma^{\mu} \ell_{L}\right. \\
& \left.-2 \bar{E}_{R i} \gamma^{\mu} E_{R i}\right)\left(\sin \xi_{Z} Z_{\mu}+\cos \xi_{Z} Z_{\mu}^{\prime}\right) \\
& -\frac{g_{Y}}{2}\left(\tan \theta_{R}+\cot \theta_{R}\right)\left(\bar{\nu}_{R \tau} \gamma^{\mu} \nu_{R \tau}-\bar{\tau}_{R} \gamma^{\mu} \tau_{R}\right) \\
& \times\left(\sin \xi_{Z} Z_{\mu}+\cos \xi_{Z} Z_{\mu}^{\prime}\right) .
\end{aligned}
$$

In this case, $\ell$ and $\ell_{L}$ are summed over $e, \mu, \tau, \nu_{e}, \nu_{\mu}, \nu_{\tau}$ and $E_{R}$ are summed over three generations.

The most interesting terms in Eqs. (9) and (10) occur in the third line and are potentially large if $\cot \theta_{R}$ is large. In the weak interaction basis they affect only the third generation whereas in the mass eigenstate basis [as written in Eq. (9)] they also give rise to flavor changing neutral currents. To satisfy the severe constraints that exist on flavor changing neutral currents we have to require that the $V_{R}^{d}$ and $V_{R}^{u}$ matrices be nearly diagonal.

In Ref. [17] we studied the case with $\xi_{Z} \neq 0$, in which $Z$ $-Z^{\prime}$ mixing is responsible for the shifts in the effective right-handed coupling of the $b$ quark. Within this scenario, the model given above also induces large shifts in the righthanded coupling of the $\tau$ lepton, making it phenomenologically unacceptable. One finds for large $\cot \theta_{R}[21]$,

$$
\begin{aligned}
& \delta g_{R b} \approx-\sin \theta_{W} \cot \theta_{R} V_{R b b}^{d *} V_{R b b}^{d} \xi_{Z} \\
& \delta g_{R t} \approx \sin \theta_{W} \cot \theta_{R} V_{R t t}^{u *} V_{R t t}^{u} \xi_{Z}, \\
& \delta g_{R \tau} \approx-\sin \theta_{W} \cot \theta_{R} \xi_{Z} .
\end{aligned}
$$

This last equation constrains the product $\cot \theta_{R} \xi_{Z}$ to be at the $10^{-3}$ level or less, whereas one would need $\cot \theta_{R} \xi_{Z} \sim 0.08$ [17] to explain $A_{F B}^{b}$ through this mechanism. Nevertheless, there are several ways around this constraint. One possibility is to eliminate the relation between the $b$-quark and $\tau$-lepton couplings to the new gauge bosons. To maintain a model that is anomaly free, this is accomplished by introducing additional fermions and can be done in more than one way. Two examples are given below. A second possibility is to require the $Z-Z^{\prime}$ mixing to be small (or zero) and in this way satisfy the constraints from $\tau$ leptons. As we discuss in Sec. III, there is a second mechanism at the loop level by which the model can induce significant shifts on $\delta g_{R b}$ and not on $\delta g_{R \tau}$.

We now discuss two ways to modify the model so that it remains anomaly free but does not have enhanced couplings for the $\tau$ lepton in the case of large $\cot \theta_{R}$

\section{A. Modified lepton sector}

In this first example we keep the quark sector as above but make some modifications to the lepton content. The lepton sector consists of the usual three generations [all transforming as in Eq. (2)] plus

$$
L_{R}=\left(\begin{array}{c}
\nu_{R}^{\prime} \\
e_{R}^{\prime}
\end{array}\right)=(1,1,2)(-1), \quad e_{L}^{\prime}=(1,1,1)(-2) .
$$

Compared with the particle content of Eqs. (2) and (3), the net new particles are $e_{L}^{\prime}$ and $E_{R}(3)$. Their contributions to gauge anomaly cancel each other, and therefore the theory is anomaly free.

The new particle $e^{\prime}$ can be made heavy because $H_{R}$ provides its mass. The neutral new particle $\nu_{R}^{\prime}$ can be made heavy by introducing a $\Delta_{R}(1,1,3)(-2)$ Higgs boson representation with large VEV. Therefore, at low energy one does not need to consider the effect of the new fermions.

The couplings for the usual three generations of leptons become,

$$
\begin{aligned}
\mathcal{L}_{Z}(\text { lepton })= & -\frac{g_{L}}{2 \cos \theta_{W}} \bar{\ell} \gamma^{\mu}\left(g_{V}-g_{A} \gamma_{5}\right) \ell\left(\cos \xi_{Z} Z_{\mu}\right. \\
& \left.-\sin \xi_{Z} Z_{\mu}^{\prime}\right)+\frac{g_{Y}}{2} \tan \theta_{R}\left(-\bar{\ell}_{L} \gamma^{\mu} \ell_{L}\right. \\
& \left.-2 \bar{E}_{R i} \gamma^{\mu} E_{R i}\right)\left(\sin \xi_{Z} Z_{\mu}+\cos \xi_{Z} Z_{\mu}^{\prime}\right) .
\end{aligned}
$$

Once again $\ell$ and $\ell_{L}$ are summed over $e, \mu, \tau, \nu_{e}, \nu_{\mu}, \nu_{\tau}$ and $E_{R}$ is summed over three generations. The couplings of the new leptons are 


$$
\begin{aligned}
\mathcal{L}_{Z}(\text { lepton })= & -\frac{g_{R}}{\sqrt{2}}\left[\bar{\nu}_{R}^{\prime} \gamma_{\mu} e_{R}^{\prime} W_{R}^{\mu}+\text { H.c. }\right]-\frac{g_{L}}{2 \cos \theta_{W}} \\
& \times\left(-2 q \sin ^{2} \theta_{W}\right) \bar{e}^{\prime} \gamma^{\mu} e^{\prime}\left(\cos \xi_{Z} Z_{\mu}-\sin \xi_{Z} Z_{\mu}^{\prime}\right) \\
& +\frac{g_{Y}}{2} \tan \theta_{R}\left(-2 \bar{e}^{\prime} \gamma^{\mu} e^{\prime}\right)\left(\sin \xi_{Z} Z_{\mu}+\cos \xi_{Z} Z_{\mu}^{\prime}\right) \\
& -\frac{g_{Y}}{2}\left(\tan \theta_{R}+\cot \theta_{R}\right)\left(\bar{\nu}_{R}^{\prime} \gamma^{\mu} \nu_{R}^{\prime}-\bar{e}_{R}^{\prime} \gamma^{\mu} e_{R}^{\prime}\right) \\
& \times\left(\sin \xi_{Z} Z_{\mu}+\cos \xi_{Z} Z_{\mu}^{\prime}\right) .
\end{aligned}
$$

\section{B. Modified quark sector}

In this case we have three generations of leptons transforming as in Eq. (2) with couplings as in Eq. (13), and we introduce additional quarks to cancel the anomalies:

$$
\begin{aligned}
Q_{L}^{\prime}=\left(\begin{array}{c}
u_{L}^{\prime} \\
d_{L}^{\prime}
\end{array}\right) & =(3,1,2)(1 / 3), \quad u_{R}^{\prime}=(3,1,1)(4 / 3), \\
d_{R}^{\prime} & =(3,1,1)(-2 / 3) .
\end{aligned}
$$

The usual three generations of quarks have the same quantum numbers as in Eqs. (2), (3) and couplings as in Eqs. (8), (9). Again the above particle content gives a gauge anomaly free theory, and the new particles can be made heavy because they receive their mass from the VEV of $H_{R}$. The new quarks have couplings

$$
\begin{aligned}
\mathcal{L}_{Z}(\text { quark })= & -\frac{g_{L}}{2 \cos \theta_{W}}\left(-2 q \sin ^{2} \theta_{W}\right) \bar{q}^{\prime} \gamma^{\mu} q^{\prime}\left(\cos \xi_{Z} Z_{\mu}\right. \\
& \left.-\sin \xi_{Z} Z_{\mu}^{\prime}\right)+\frac{g_{Y}}{2} \tan \theta_{R}\left(\frac{4}{3} \bar{u}^{\prime} \gamma^{\mu} u^{\prime}-\frac{2}{3} \bar{d}^{\prime} \gamma^{\mu} d^{\prime}\right) \\
& \times\left(\sin \xi_{Z} Z_{\mu}+\cos \xi_{Z} Z_{\mu}^{\prime}\right)-\frac{g_{Y}}{2}\left(\tan \theta_{R}+\cot \theta_{R}\right) \\
& \times\left(\bar{u}_{L}^{\prime} \gamma^{\mu} u_{L}^{\prime}-\bar{d}_{L}^{\prime} \gamma^{\mu} d_{L}^{\prime}\right)\left(\sin \xi_{Z} Z_{\mu}+\cos \xi_{Z} Z_{\mu}^{\prime}\right) .
\end{aligned}
$$

\section{Discussion}

The previous two examples illustrate how it is possible to single out the $b$ and $t$ quarks with a new right-handed interaction without affecting the $\tau$ lepton very much. The price paid is, of course, the introduction of additional fermions. The additional fermions can be made heavy and this allows us to ignore them at this stage, where we are interested only in the effect of potentially strong right-handed couplings of the $b$ and $t$ quarks in LEP observables. The new heavy fermions are only used to illustrate that it is possible to construct a renormalizable, anomaly free, model of this type.

The couplings of the $b$ quark to the new right-handed gauge bosons remain as in the original model so that, according to Eq. (11), we require $\xi_{Z} \cot \theta_{R} \sim 0.08$ to explain $A_{F B}^{b}$ [17] through $Z-Z^{\prime}$ mixing. With $\cot \theta_{R}$ large, the new phys- ics effects mainly affect the third generation of quarks; our model is in some sense "leptophobic."

In Ref. [17], we pointed out that the process $b \rightarrow s \gamma$ severely constrains the mixing of the charged gauge bosons $\xi_{W}$. This constraint is not in conflict with the mixing needed in the neutral sector $\xi_{Z}$ to fit $A_{F B}^{b}$ as discussed in Ref. [17]. Here we point out that there is another way to obtain Eq. (11) without affecting $b \rightarrow s \gamma$. This involves a new model in which the $S U(2)_{R}$ is replaced by a $U(1)_{R}$ with up quarks (leptons) and down quarks (leptons) in $S U(2)_{R}$ doublets carrying 1 and -1 of $U(1)_{R}$ charges, respectively. This model will also give $\delta g_{R b} \sim \xi_{Z} \cot \theta_{R}$ as in Eq. (11), but it now arises in the context of models where (a) there are no large contributions to $Z \rightarrow \tau^{+} \tau^{-}$, and (b) there are no new charged gauge bosons $W^{\prime}$, so that there are no constraints from $b$ $\rightarrow s \gamma$. The contributions to the parameter $T$ that occur through mixing of the $Z$ and $Z^{\prime}$ are identical to Ref. [17] and lead to the allowed region of Fig. 1 in that reference.

The most important new feature common to all the models that we have discussed is the existence of a new $Z^{\prime}$ gauge boson which has enhanced couplings to top and bottom quarks (and perhaps to the $\tau$ lepton provided its mixing with the $Z$ is sufficiently small). In Sec. IV we explore the bounds that exist on the mass of this $Z^{\prime}$ from LEP-II measurements.

\section{ONE-LOOP CONTRIBUTIONS TO $\delta g_{R b}$}

In models such as the ones presented in Sec. II, with a new $S U(2)_{R}$ gauge interaction, there is a one-loop contribution to $\delta g_{R b}$ that is present even when there is no mixing. A priori we can expect this contribution to be similar in size to the standard model contribution to $\delta g_{L b}$ proportional to $M_{t}^{2}$. One can imagine a suppression of the form $\left(M_{W} / M_{W_{R}}\right)^{2}$ with respect to the standard model $\delta g_{L b}$, but this can be compensated by an enhancement $\left(g_{R} / g_{L}\right)^{2} \sim \cot ^{2} \theta_{R}$ in the right-handed gauge couplings.

It would be impossible to present a complete one-loop calculation for $Z \rightarrow b \bar{b}$ in the general case of Sec. II because we do not have sufficient information at present to determine all the parameters in those models. At the same time, we are interested more in exploring the idea of a potentially strong right-handed interaction affecting the $b$ and $t$ quarks than in the specific details of the models in Sec. II. For this reason, we consider a slightly simpler calculation that has the ingredients we need. First, we will only concern ourselves with the one-loop corrections that are enhanced by $\left(g_{R} / g_{L}\right)^{2}$ with respect to one-loop electroweak corrections. Second, we will require that there be no $Z-Z^{\prime}$ nor $W-W^{\prime}$ mixing in the model. Finally, we will treat all standard model fermions as massless except for the top quark.

\section{A. Model with no tree-level mixing}

To eliminate the tree-level mixing in the models of Sec. II in a simple manner we first require $v_{2}=0$ in Eq. (5). This immediately makes $\xi_{W}=0$ and allows us to simplify the notation by calling the remaining VEV in $\phi v \equiv v_{1}$. We further make $\xi_{Z}=0$ at the tree level by imposing the condition 


$$
v_{L}=v \cot \theta_{R}
$$

in Eq. (5). The parameter $\xi_{Z}$ describing the $Z-Z^{\prime}$ mixing is the only one (beyond those already appearing in the standard model) that enters the result for the $Z \rightarrow b \bar{b}$ partial width at the tree level. As such, it is the only new parameter that needs to be defined at one-loop in our calculation of $Z$ $\rightarrow b \bar{b}$, and we will return to this point at the end of the section.

In the simplified model, the gauge boson masses become

$$
\begin{gathered}
M_{W}^{2}=\frac{g_{L}^{2}}{2}\left(v_{L}^{2}+v^{2}\right)=\frac{g_{R}^{2}}{2 \tan ^{2} \theta_{W}} v^{2}, \quad M_{Z}^{2}=\frac{M_{W}^{2}}{\cos ^{2} \theta_{W}} \\
M_{W_{R}}^{2}=\frac{g_{R}^{2}}{2}\left(v_{R}^{2}+v^{2}\right), \quad M_{Z_{R}}^{2}=\frac{g_{R}^{2}}{2}\left(\frac{v_{R}^{2}}{\cos ^{2} \theta_{R}}+v^{2}\right)
\end{gathered}
$$

so that $M_{W_{R}} \approx M_{Z_{R}}$ for large $\cot \theta_{R}$ (and equal to $M_{W^{\prime}}$ and $M_{Z}$, since in this case there is no mixing). Recalling the relation $g_{R} \sin \theta_{R}=g_{L} \tan \theta_{W}$, we see that this sector of the model is characterized by the two ratios of VEV's,

$$
\frac{v_{L}}{v}=\cot \theta_{R}, \quad \frac{v_{R}}{v} \approx \frac{M_{W_{R}}}{M_{W} \tan \theta_{W}}
$$

where the last expression follows for $v_{R} / v \gg 1$. It will also be convenient to define $x_{v}=v^{2} /\left(v^{2}+v_{R}^{2}\right)$.

In the approximation $m_{b}=0$, the Yukawa Lagrangian necessary to generate the top-quark mass is given by

$$
\mathcal{L}_{Y}=-\kappa\left(\begin{array}{ll}
\bar{t}_{L} & \bar{b}_{L}
\end{array}\right) \phi\left(\begin{array}{c}
t_{R} \\
b_{R}
\end{array}\right)+\text { H.c. }
$$

All the couplings in this Yukawa potential can thus be written in terms of $m_{t}$ and $v$ as in Eq. (A4).

\section{B. Loops with right-handed gauge bosons}

We are ready to calculate the one-loop corrections to $\delta g_{R b}$ that are enhanced by $\cot ^{2} \theta_{R}$. We start by considering the diagrams in Fig. 1 that do not involve scalar mesons in the loop. We work in unitary gauge with the vertices given in the Appendix, and we use dimensional regularization with the notation

$$
\frac{1}{\epsilon}=\frac{2}{4-n}-\gamma+\log (4 \pi)-\log \mu^{2} .
$$

Relegating details for each diagram to the Appendix, we can write a simple analytical result for the sum of the diagrams in Fig. 1 in the limit $M_{Z}=0$,
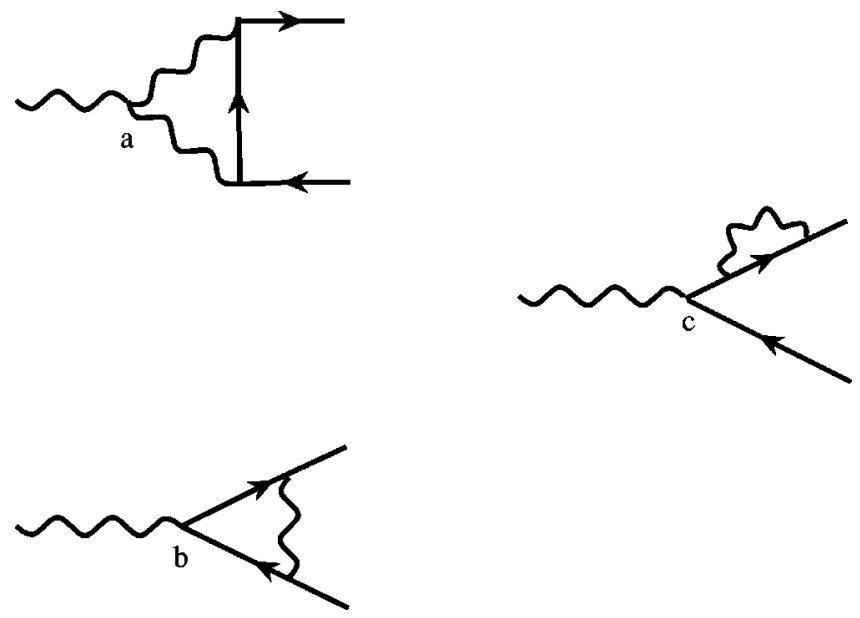

FIG. 1. Unitary gauge diagrams for $Z \rightarrow b \bar{b}$ that do not involve scalars.

$$
\begin{aligned}
\left(\delta g_{R b}\right)_{G B}= & \frac{g_{R}^{2}}{16 \pi^{2}} \frac{M_{t}^{2}}{M_{W_{R}}^{2}} \frac{1}{4}\left[\frac{1}{\epsilon}-\frac{7}{2}-\log \left(\frac{M_{t}^{2}}{\mu^{2}}\right)\right. \\
& \left.-3 \log \left(\frac{M_{t}^{2}}{M_{W_{R}}^{2}}\right)\right] .
\end{aligned}
$$

Later on we will show numerical results for $M_{Z} \neq 0$. Unlike the counterpart of this calculation in the standard model, Eq. (28), Eq. (22) is divergent. This indicates the presence of additional contributions to this process in our model.

There are two additional diagrams of the form of Fig. 1 that give corrections to the right-handed coupling and that are enhanced by $g_{R}^{2}$. They look like Figs. 1(b) and 1(c) with an exchange of a $Z_{R}$ (and therefore $b$ quarks in the intermediate lines). Both of these turn out to be finite and their finite parts precisely cancel each other out in the $M_{Z}=0$ limit.

\section{Loops with scalars}

We consider next the contributions from diagrams in which scalars appear in the loops, as in Fig. 2.
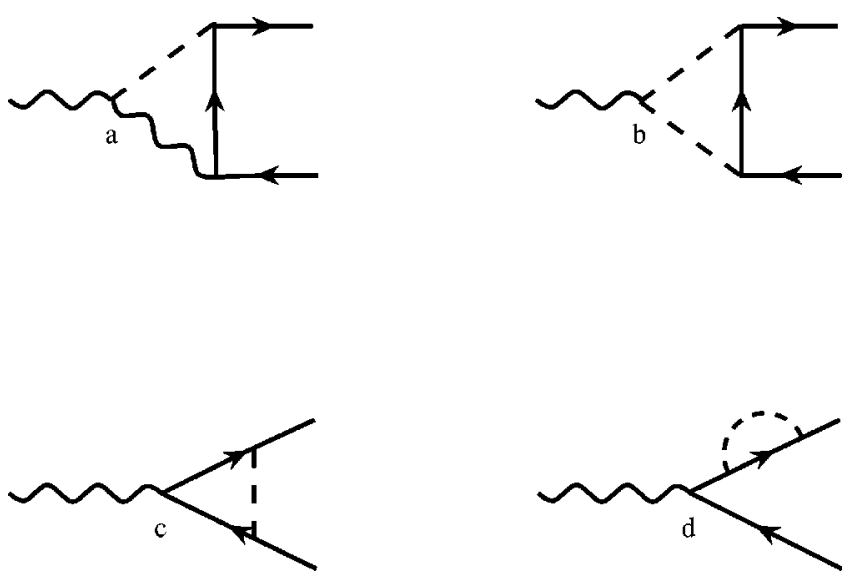

FIG. 2. $Z \rightarrow b \bar{b}$ one-loop diagrams involving scalars. 


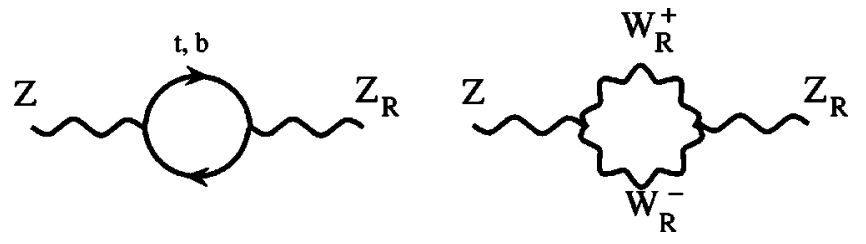

FIG. 3. $Z-Z^{\prime}$ mixing at one-loop.

The finite part that results from these diagrams is model dependent. In particular it depends on the details of the scalar potential, which we have not specified, and which determines the masses of the physical scalar and pseudo-scalar mesons present in the model. We are only interested here in estimating the size of the vertex corrections in Fig. 1, and wish to consider the diagrams in Fig. 2 only insofar as they are needed to render the result finite. For this purpose it is sufficient to identify a basis for the scalars that is orthogonal to the would-be Goldstone bosons that give the gauge bosons their mass. We consider all physical scalars to be degenerate and to have a large mass, of the order of $M_{W_{R}}$. With details relegated to the Appendix, we find that the sum of these diagrams contributes the following terms that are enhanced by $\cot ^{2} \theta_{R}$ :

$$
\begin{aligned}
\left(\delta g_{R b}\right)_{S} & =\frac{1}{16 \pi^{2}}\left(\frac{m_{t}}{v}\right)^{2}\left(-\frac{1}{2} x_{v}\left(1-x_{v}\right)\right)\left[\frac{1}{\epsilon}-\log \left(\frac{M_{H}^{2}}{\mu^{2}}\right)+\frac{5}{2}\right] \\
& =\frac{g_{R}^{2}}{16 \pi^{2}} \frac{M_{t}^{2}}{M_{W_{R}}^{2}} \frac{1}{4}\left[-\frac{1}{\epsilon}+\log \left(\frac{M_{H}^{2}}{\mu^{2}}\right)-\frac{5}{2}\right] .
\end{aligned}
$$

Notice that the left-handed coupling $\delta g_{L b}$ does not receive corrections from the sum of diagrams in Fig. 2. With this result, Eq. (23), we find that the divergent terms precisely cancel the leftover ones from the gauge boson sector in Eq. (22) leaving us with a finite answer.

\section{Renormalization and $Z-Z^{\prime}$ mixing}

Finally we comment on the renormalization scheme used. At the tree level, the $Z \rightarrow b \bar{b}$ decay width (or $A_{F B}^{b}$ ) takes the same value as in the standard model in the absence of $Z$ $-Z^{\prime}$ mixing. We can therefore express it in terms of the input parameters $G_{F}$, the physical $Z$ mass and $\alpha\left(M_{Z}\right)$ as is usually done for the standard model case. It is clear from the vertices given in the Appendix, Sec. 3, that none of these quantities receives one-loop corrections that are enhanced by $\cot ^{2} \theta_{R}$. The only input parameter that receives enhanced corrections is the $Z-Z^{\prime}$ mixing angle $\xi_{Z}$ through diagrams such as those in Fig. 3. These diagrams (and a few others) have an enhancement of $\cot \theta_{R}$ through the $Z^{\prime} t \bar{t}$ or $Z^{\prime} b \bar{b}$ coupling in the first case and through the $W_{R}^{+} W_{R}^{-} Z^{\prime}$ coupling in the second case. When the $Z^{\prime}$ line is connected to $b \bar{b}$, a second $\cot \theta_{R}$ factor is picked up leading to corrections in $Z \rightarrow b \bar{b}$ that are enhanced by $\cot ^{2} \theta_{R}$. In view of this, our simplest option is to adopt a renormalization scheme in which $\xi_{Z}$ $\equiv 0$ at one loop. That is, we absorb the corrections from Fig. 3 into the definition of $\xi_{Z}$. This completes the discussion of all the one-loop corrections needed to yield a finite $\delta g_{R b}$ and we now turn our attention to its possible size.

\section{E. Numerical results}

Adding the results from all diagrams discussed above, we find in the $M_{Z}=0$ limit,

$$
\delta g_{R b}=\frac{g_{R}^{2}}{16 \pi^{2}} \frac{M_{t}^{2}}{M_{W_{R}}^{2}} \frac{1}{4}\left[-6+3 \log \left(\frac{M_{W_{R}}^{2}}{M_{t}^{2}}\right)+\log \left(\frac{M_{H}^{2}}{M_{t}^{2}}\right)\right] \text {. }
$$

To illustrate the magnitude of this correction consider the case

$$
\cot \theta_{R}=\frac{v_{L}}{v} \approx \frac{v_{R}}{v} \sim 10
$$

which implies

$$
\frac{g_{R}^{2}}{M_{W_{R}}^{2}} \approx \frac{g_{L}^{2}}{M_{W}^{2}}
$$

and therefore

$$
\delta g_{R b}=\frac{\sqrt{2} G_{F} M_{t}^{2}}{8 \pi^{2}}\left[-3+\frac{3}{2} \log \left(\frac{M_{W_{R}}^{2}}{M_{t}^{2}}\right)+\frac{1}{2} \log \left(\frac{M_{H}^{2}}{M_{t}^{2}}\right)\right] .
$$

This is to be compared with the corresponding correction to $\delta g_{L b}$ in the standard model which is given by [18]

$$
\delta g_{L b}=\frac{\sqrt{2} G_{F} M_{t}^{2}}{8 \pi^{2}} .
$$

This shows that with large $\cot \theta_{R}$ as in Eq. (25), $\delta g_{R b}$ in our model is of the same order as the one-loop correction to $\delta g_{L b}$ proportional to $M_{t}^{2}$ in the standard model.

In order to include kinematic effects from $M_{Z} \neq 0$, we compute the integrals over Feynman parameters numerically. It is convenient to present the result in the form

$$
\delta g_{R b}=\frac{\sqrt{2} G_{F} M_{t}^{2}}{8 \pi^{2}} F_{1}\left(M_{W_{R}}\right)
$$

for the case $g_{L}^{2} / M_{W}^{2} \approx g_{R}^{2} / M_{W_{R}}^{2}$. We show $F_{1}$ in Fig. 4(a). In the more general case it is convenient to write

$$
\delta g_{R b}=\frac{g_{R}^{2}}{32 \pi^{2}} F_{2}\left(M_{W_{R}}\right)
$$

and we show $F_{2}$ in Fig. 4(b). These results indicate that a contribution to $\delta g_{R b}$ at the percent level is possible in models with $\cot \theta_{R} \sim 10$. 

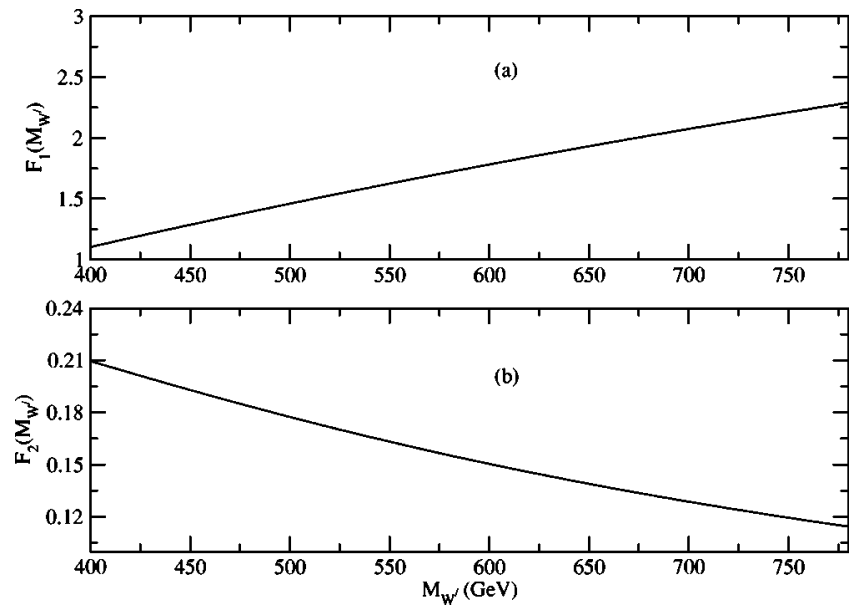

FIG. 4. Form factors $F_{1}\left(M_{W_{R}}\right)$ and $F_{2}\left(M_{W_{R}}\right)$ evaluated numerically to include kinematic effects from a non-zero $M_{Z}$ for fixed $M_{H}=700 \mathrm{GeV}$.

\section{CONSTRAINTS FROM LEP-II}

The mass of additional $Z^{\prime}$ gauge bosons that occur in many models is constrained to be larger than about $500 \mathrm{GeV}$ [2]. These bounds arise mostly from processes involving four first or second generation fermions and do not apply to nonuniversal $Z^{\prime}$ gauge bosons that couple strongly to third generation fermions but very weakly to first and second generation fermions. Roughly speaking, when a $Z^{\prime}$ like this is exchanged in a process such as $b \bar{b} \rightarrow b \bar{b}$ it generates an amplitude of the order of electroweak strength times $\cot ^{2} \theta_{R}$. For the models that we have in mind $\cot \theta_{R} \sim 10$ this can be a very significant enhancement. On the other hand when the same $Z^{\prime}$ is exchanged between fermions of the first two generations, in processes such as $u \bar{u} \rightarrow u \bar{u}$, it generates an amplitude of electroweak strength times $\tan ^{2} \theta_{R}$ which is drastically suppressed.

The best bounds one can have at present on such a $Z^{\prime}$ come from a process in which a first or second generation fermion pair produces a $b \bar{b}$ pair. Since $b \bar{b}$ production in hadron colliders is mostly a strong interaction process, the most promising reaction to constrain our $Z^{\prime}$ is $e^{+} e^{-} \rightarrow b \bar{b}$ studied at LEP-II. Notice that for a process such as this one, the exchange of a $Z^{\prime}$ results in a correction of electroweak strength, suppressed only by the mass of the $Z^{\prime}$. The cross section for this process is largely independent of the value of $\cot \theta_{R}$. At leading order, $\cot \theta_{R}$ only appears through the width of the $Z^{\prime}$ that one must include in the propagator for $s$-channel exchange.

In this section we use the LEP-II data on $e^{+} e^{-} \rightarrow b \bar{b}$ and $e^{+} e^{-} \rightarrow \tau^{+} \tau^{-}$to constrain the mass of these non-universal $Z^{\prime}$ gauge bosons. The calculation is performed numerically using the program COMPHEP [22] with the following strategy. We use COMPHEP to calculate tree level cross sections for $e^{+} e^{-} \rightarrow f \bar{f}$ at LEP-II energy both for the standard model and for the standard model plus the $Z^{\prime}$ of Sec. II. We then use these cross sections to construct the ratios $R_{b} /\left(R_{b}\right)_{S M \text {-tree }}$ and similarly for $A_{F B}^{b}$. We then compare these ratios to the

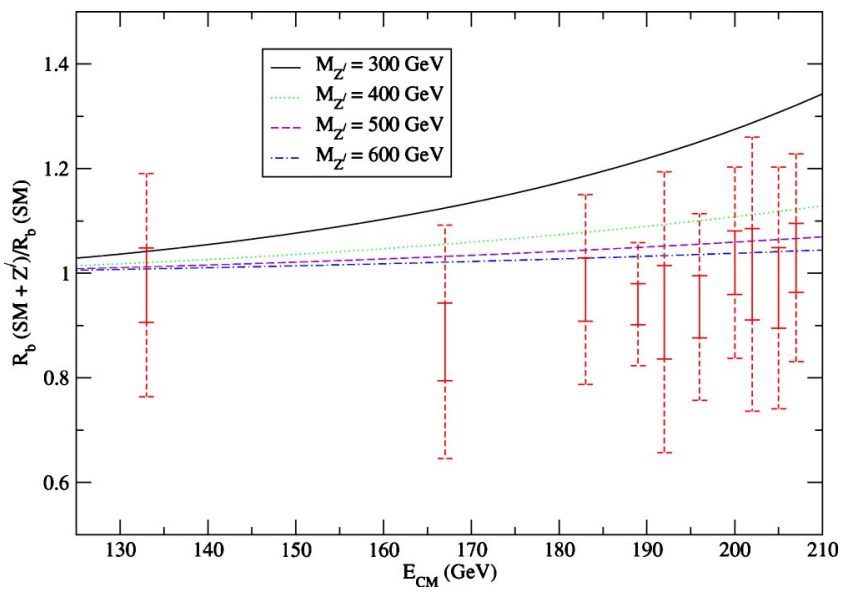

FIG. 5. $R_{b}$ at LEP-II energies for $\cot \theta_{R}=15$ with no $Z-Z^{\prime}$ mixing. The different curves correspond to $M_{Z^{\prime}}$ of $300,400,500$ and $600 \mathrm{GeV}$. The data points from Ref. [1] are shown with their $1-\sigma$ and $3-\sigma$ error bars.

corresponding ratios $\left(R_{b}\right)_{E X P} /\left(R_{b}\right)_{S M}$ where $\left(R_{b}\right)_{E X P}$ are the averages of LEP-II measurements as reported in Ref. [1] and $\left(R_{b}\right)_{S M}$ is the full standard model expectation computed with ZFITTER as reported in Table 8.7 of Ref. [1]. Schematically for the cross section,

$$
\begin{aligned}
\frac{\sigma_{\text {Theory }}}{\sigma_{S M}} & =\frac{\sigma_{S M \text {-tree }}+\sigma_{S M \text {-loop }}+\sigma_{Z^{\prime}}}{\sigma_{S M \text {-tree }}+\sigma_{S M-\text { loop }}} \approx \frac{\sigma_{S M \text {-tree }}+\sigma_{Z^{\prime}}}{\sigma_{S M \text {-tree }}} \\
& =1+\frac{\sigma_{Z^{\prime}}}{\sigma_{S M \text {-tree }}} .
\end{aligned}
$$

In this way the error that results from our using only the tree-level result for the standard model prediction from COMPHEP becomes higher order in our comparison with data.

In models with $Z-Z^{\prime}$ mixing, we need to remove the enhanced coupling $Z^{\prime} \tau^{+} \tau^{-}$as discussed in the previous section. In that case the only relevant LEP-II process to bound the $Z^{\prime}$ is $e^{+} e^{-} \rightarrow b \bar{b}$. There are two observables that can be used: $R_{b}$ and $A_{F B}^{b}$. In Fig. 5 we show $R_{b} /\left(R_{b}\right)_{S M}$ for different values of $M_{Z}^{\prime}$. In this figure we have assumed no mixing $\left(\xi_{Z}=0\right)$ and used $\cot \theta_{R}=15$ [23]. The LEP-II data points are shown with their $1-\sigma$ and $3-\sigma$ error bars. It is evident already from this figure that $M_{Z^{\prime}}$ will be constrained to be larger than about $500 \mathrm{GeV}$.

In Fig. 6 we show similar results for the forwardbackward asymmetry. It is evident from this figure that $A_{F B}^{b}$ does not constrain the $Z^{\prime}$ as much as $R_{b}$ does due to its larger experimental error (in this case we only show the 1- $\sigma$ error bars).

As discussed in Sec. II, it is possible to allow $Z-Z^{\prime}$ mixing in models where the couplings to $\tau^{ \pm}$are not enhanced. We illustrate the effect of including this mixing in Fig. 7. For each value of $M_{Z^{\prime}}$, we have allowed $\xi_{Z}$ to vary between zero and $\xi_{Z}= \pm 0.08 / \cot \theta_{R}$, the value required to fit $A_{F B}^{b}$ from LEP-I. We see that mixing is a small effect on $R_{b}$ at LEP-II energies. 


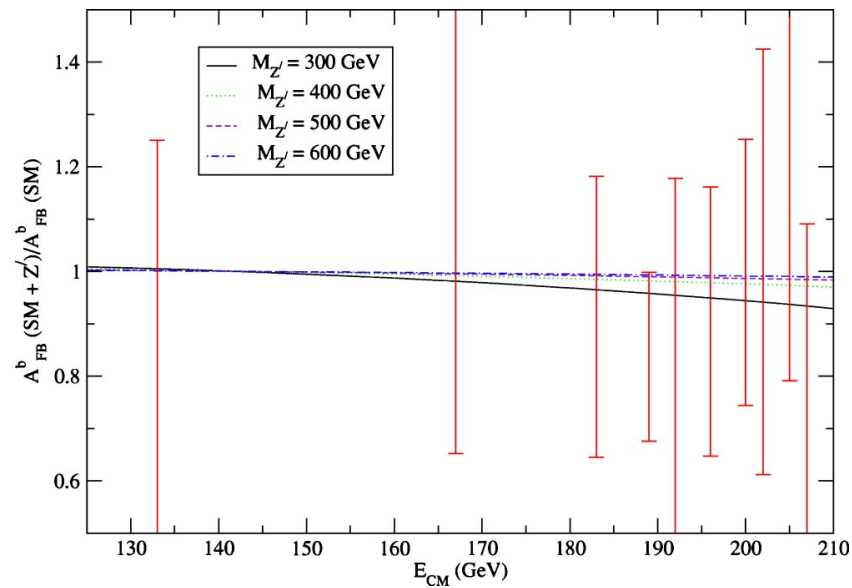

FIG. 6. Same as Fig. 5 for $A_{F B}^{b}$. Only the $1-\sigma$ error bars are shown for the data points from Ref. [1].

In Fig. 8 we illustrate the effect of varying $\cot \theta_{R}$ and thus the $Z^{\prime}$ width for $M_{Z^{\prime}}=400 \mathrm{GeV}$. We use values $\cot \theta_{R}$ $=10,15$ and also show the result of approximating the $Z^{\prime}$ exchange with a contact interaction. The results illustrate that below the resonance, the bound on the $Z^{\prime}$ mass becomes slightly tighter for narrower resonances $\left(\right.$ smaller $\cot \theta_{R}$ ). In our model, the interference between $Z$ and $Z^{\prime}$ exchange amplitudes is always constructive in the energy region between the two resonances. The figure also illustrates that for a $Z^{\prime}$ as light as $400 \mathrm{GeV}$, a contact interaction is a reasonable approximation for effects at LEP-II energies. We shall use this later when comparing our bounds with those extracted by the LEP-II analysis group for contact interactions.

To quantify the bounds on the $Z^{\prime}$ mass we construct a $\chi^{2}$ for a fit to LEP-II data with our model. In Fig. 9 we present this result after subtracting the $\chi^{2}$ from a standard model fit (using the ZFITTER results quoted in Ref. [1]). Once again we show the three cases $\cot \theta_{R}=10,15$ and a contact interaction

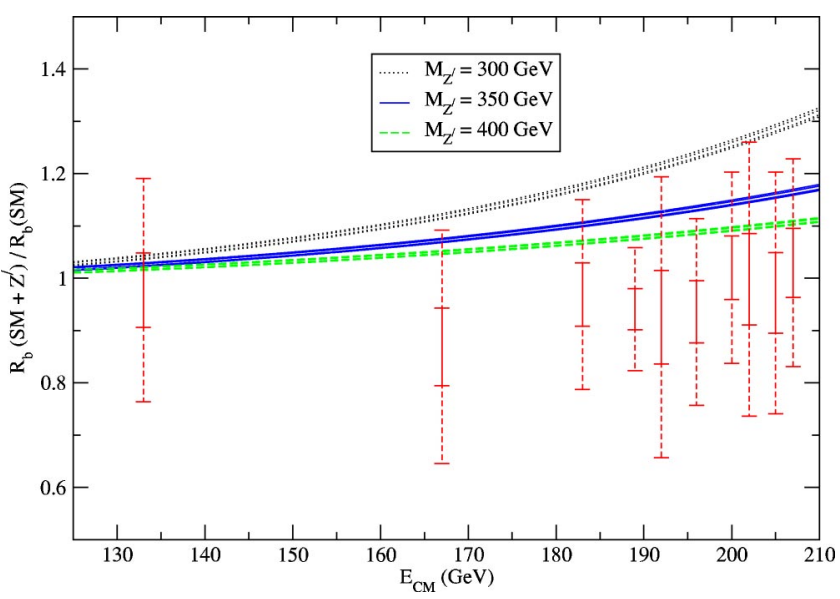

FIG. 7. Same as Fig. 5 but allowing for $Z-Z^{\prime}$ mixing. The bands shown correspond to $\cot \theta_{R} \xi_{z}$ ranging from 0 to \pm 0.08 with $\cot \theta_{R}=15$. For each mass, the upper end of the band corresponds to $\xi_{Z}=-0.005$ and the lower end of the band corresponds to $\xi_{Z}$ $=0.005$. Once again the data points are from Ref. [1].

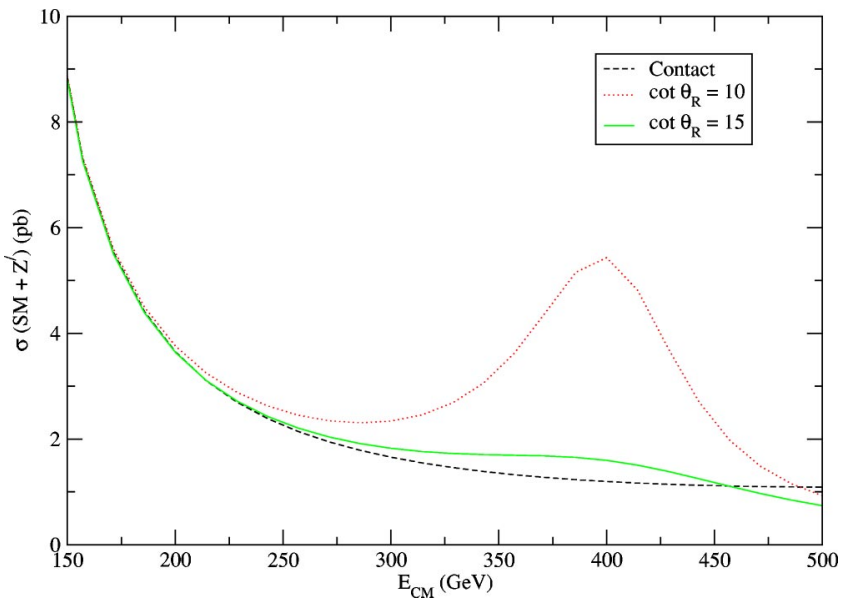

FIG. 8. Cross section for $e^{+} e^{-} \rightarrow b \bar{b}$ with $M_{Z^{\prime}}=400 \mathrm{GeV}$ for $\cot \theta_{R}=10,15$ and for a contact interaction approximating the $Z^{\prime}$ exchange amplitude.

approximation. It is important to notice, for example in Fig. 5, that the LEP-II data are consistently below the standard model prediction. This, combined with the fact that the interference between the $Z$ and $Z^{\prime}$ amplitudes in our model is always constructive in this energy region, implies that the standard model is always a better fit than any of our $Z^{\prime}$ models. If we require that the new model does not deviate from the standard model by 2(3) standard deviations, we can place the bounds $M_{Z^{\prime}}>700(540) \mathrm{GeV}$ for $\cot \theta_{R}=15$. Given that the LEP-II data are consistently below the standard model expectation, it is conceivable that there is some common systematic error not accounted for in the quoted error bars. To account for this possibility, we naively rescale the data by a common factor in such a way as to minimize the $\chi^{2}$ of the standard model fit. Doing this results in lower bounds on the $Z^{\prime}$ mass. For example, for $\cot \theta_{R}=10$ the 2(3) sigma bounds move from 780 (595) $\mathrm{GeV}$ to 530 (460) GeV.

We can also use the contact interaction approximation to bound the $Z^{\prime}$ mass. The correspondence to Table 8.12 of Ref. $[1]$ is (for no mixing)

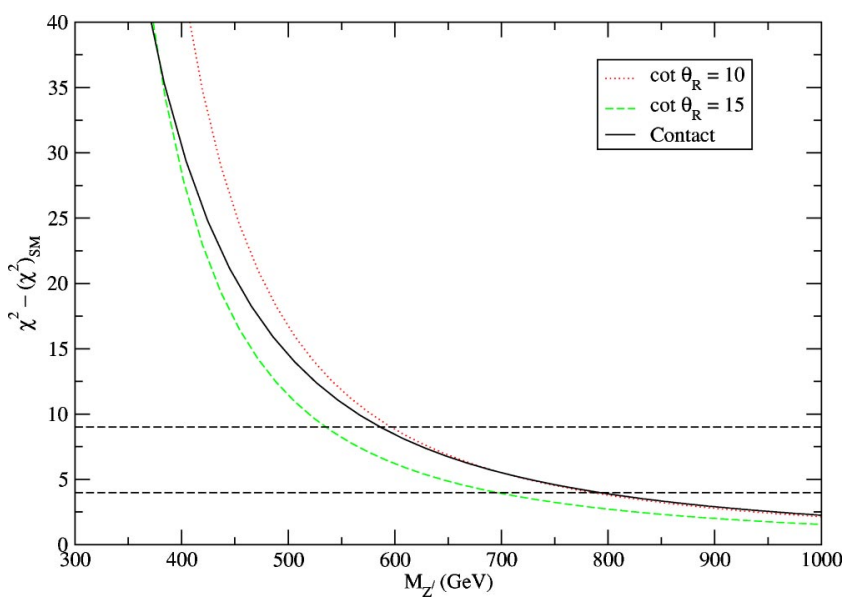

FIG. 9. $\chi^{2}-\chi_{S M}^{2}$ for fits to $R_{b}$ at LEP-II energies as a function of $M_{Z^{\prime}}$ setting $\xi_{Z}=0$. 


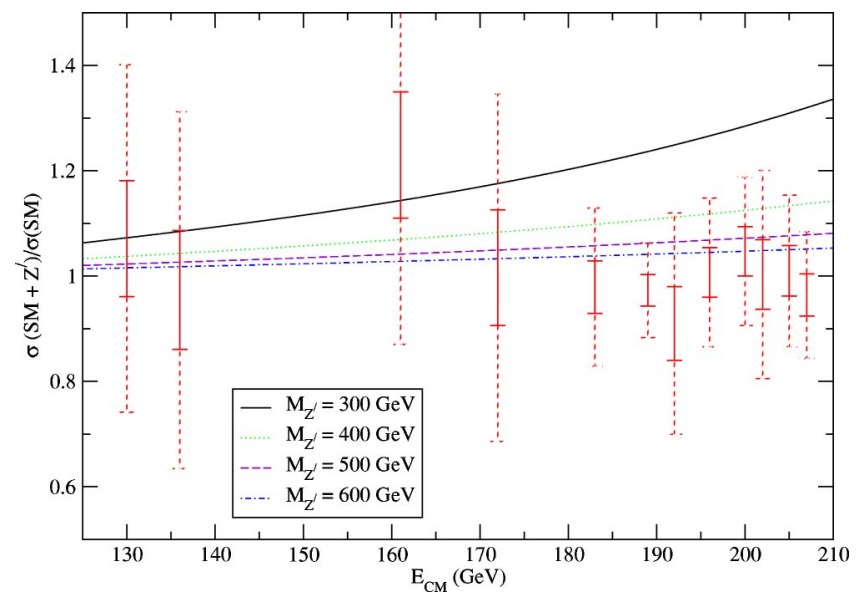

FIG. 10. $\sigma\left(e^{+} e^{-} \rightarrow \tau^{+} \tau^{-}\right)$for $\cot \theta_{R}=15$. The data points are from Ref. [1].

$$
M_{Z^{\prime}}=\sqrt{\eta} \frac{g_{L} \tan \theta_{W}}{4 \sqrt{\pi}} \Lambda .
$$

Our model of Sec. II generates both a $L R$ contact interaction with $\eta_{L R}=1$ and a $R R$ contact interaction with $\eta_{R R}=2$. For constructive interference we thus infer the $95 \%$ confidence level bounds $M_{Z^{\prime}}>544 \mathrm{GeV}$ from the $R R$ interaction and $M_{Z^{\prime}}>275 \mathrm{GeV}$ from the $L R$ interaction.

From all this we conclude that in all cases the $Z^{\prime}$ is already restricted to be heavier than about $500 \mathrm{GeV}$ by LEP-II data.

Finally, since models without mixing also allow large couplings to the $\tau$ lepton, we show in Fig. 10 the crosssection for $e^{+} e^{-} \rightarrow \tau^{+} \tau^{-}$at LEP-II. A calculation of the $\chi^{2}$ for the fit in this case indicates that the bounds on the $Z^{\prime}$ mass are slightly higher than those obtained from studying $R_{b}$, but not significantly so.

\section{CONCLUSIONS}

Motivated by the 3- $\sigma$ discrepancy between the standard model prediction and the measured forward-backward asymmetry $A_{F B}^{b}$ at the $Z$ peak we have studied models which can generate a sufficiently large $\delta g_{R b}$ through new non-universal right-handed gauge interactions.

One possible mechanism to generate this $\delta g_{R b}$ is the mixing of the $Z$ with a $Z^{\prime}$. We had already discussed a model such as this in Ref. [17]. In this paper we have illustrated several variations on that model that are also renormalizable and anomaly free. At the cost of introducing additional fermions, we showed two models that produce the required $\delta g_{R b}$ while satisfying the LEP constraints on $\delta g_{R \tau}$. We have also indicated how it is possible to modify these models so that they are not constrained by $b \rightarrow s \gamma$.

We have identified a second mechanism to generate $\delta g_{R b}$ even in cases with no $Z-Z^{\prime}$ mixing. This occurs in models with an $S U(2)_{R}$ triplet of gauge bosons at one-loop, and can give rise to $\delta g_{R b}$ at the $1 \%$ level. By itself, this mechanism is not sufficient to explain the full $\delta g_{R b} \sim 0.04$ favored by the data. The simple model used to illustrate this effect provides an example of a renormalizable model that can give rise to relatively large new interactions involving only the $b$ and $t$ quarks while respecting low energy constraints.

Finally we have used the LEP-II data for the process $e^{+} e^{-} \rightarrow b \bar{b}$ to place bounds on the mass of the $Z^{\prime}$ in our models. This is a $Z^{\prime}$ that couples weakly (by a factor $\tan \theta_{R} \sim 1 / 10$ weaker than standard electroweak couplings) to fermions of the first two generations. For this reason, standard bounds on $Z^{\prime}$ gauge bosons do not apply. We find that the LEP-II data constrain it to be heavier than about 500 $\mathrm{GeV}$ in all cases.

The contribution of the new gauge bosons in our models to the process $e^{+} e^{-} \rightarrow b \bar{b}$ is of electroweak strength because the enhancement in the $Z^{\prime} b \bar{b}$ coupling is compensated by the suppression in the $Z^{\prime} e^{+} e^{-}$coupling. In this way, our model is an example of a kind of new interactions that will only show their full strength in processes involving four third generation fermions. It may be possible for the LHC to study certain processes of this type, and we are currently investigating this possibility.

\section{ACKNOWLEDGMENTS}

The work of X.-G.H. was supported in part by National Science Council under grant NSC 91-2112-M-002-042, and in part by the Ministry of Education Academic Excellence Project 89-N-FA01-1-4-3. The work of G.V. was supported in part by DOE under contract No. DE-FG02-01ER41155. G.V. thanks the Department of Physics at the National Taiwan University for their hospitality while part of this work was completed.

\section{APPENDIX: VERTICES AND ONE-LOOP RESULTS}

\section{Basic conventions}

The general conventions adopted are

$$
\begin{aligned}
g_{L t} & =1-\frac{4}{3} \sin ^{2} \theta_{W}, \quad g_{R t}=-\frac{4}{3} \sin ^{2} \theta_{W}, \\
g_{L b} & =-1+\frac{2}{3} \sin ^{2} \theta_{W}, \quad g_{R b}=\frac{2}{3} \sin ^{2} \theta_{W}, \\
\Gamma_{\mu} & =-i \frac{g}{2 \cos \theta_{W}} \gamma_{\mu}\left[\left(g_{L b}+\delta g_{L b}\right) P_{L}+\left(g_{R b}+\delta g_{R b}\right) P_{R}\right] \\
\frac{1}{\epsilon} & =\frac{2}{4-n}-\gamma+\log (4 \pi)-\log \mu^{2} .
\end{aligned}
$$

\section{Scalar sector}

We start with the following parametrization for the scalars: 


$$
\begin{aligned}
H_{L} & =\left(\begin{array}{c}
\frac{h_{L}-i \phi_{L}}{\sqrt{2}}+v_{L} \\
\phi_{L}^{-}
\end{array}\right), \quad H_{R}=\left(\begin{array}{c}
\frac{h_{R}-i \phi_{R}}{\sqrt{2}}+v_{R} \\
\phi_{R}^{-}
\end{array}\right), \\
\phi & =\left(\begin{array}{cc}
\frac{h_{1}-i \phi_{1}^{0}}{\sqrt{2}}+v_{1} & \phi_{1}^{+} \\
\phi_{2}^{-} & \frac{h_{2}-i \phi_{2}^{0}}{\sqrt{2}}+v_{2}
\end{array}\right) .
\end{aligned}
$$

This parametrization contains both the would-be Goldstone bosons that give mass to the $W, Z, W_{R}, Z_{R}$ and the remaining physical scalar (or pseudo-scalar) particles.

Since we do not specify a scalar potential, we cannot identify the scalar mass eigenstates. Rather we work with a basis of physical scalars chosen to be orthogonal to the would-be Goldstone bosons, under the simplifying assumption that they all have the same large mass. The physical scalars defined this way are

$$
\begin{aligned}
& H_{1}^{ \pm}=\frac{1}{\sqrt{v^{2}+v_{L}^{2}}}\left(-v \phi_{L}^{ \pm}+v_{L} \phi_{2}^{ \pm}\right) \\
& H_{2}^{ \pm}=\frac{1}{\sqrt{v^{2}+v_{R}^{2}}}\left(-v \phi_{R}^{ \pm}+v_{R} \phi_{1}^{ \pm}\right) \\
& H_{2}^{0}=\frac{x}{\sqrt{1+x^{2}}}\left(-\sin \theta_{R} \phi_{L}^{0}+\frac{1}{x} \phi_{R}^{0}+\cos \theta_{R} \phi_{1}^{0}\right) \\
& H_{1}^{0}=\phi_{2}^{0}
\end{aligned}
$$

with $x=v_{R} /\left(v \cos \theta_{R}\right)$ with $H_{1,2}^{0}$ being neutral pseudoscalars. The only neutral scalar that enters the calculation is the original $h_{2}$. Working with this basis we can identify the divergent contributions arising from diagrams with scalar exchange (they are independent of scalar masses). The finite contributions that depend on the masses of the different scalars can only be obtained after fixing all their masses.

\section{Feynman rules}

In unitary gauge, the vertices $\gamma W_{L}^{+} W_{L}^{-}, \gamma \gamma W_{L}^{+} W_{L}^{-}$, $\gamma Z W_{L}^{+} W_{L}^{-}, Z W_{L}^{+} W_{L}^{-}$and $Z Z W_{L}^{+} W_{L}^{-}$are as in the standard model. The analogous vertices with $W_{R}^{ \pm}$taking the place of $W_{L}^{ \pm}$can be obtained by multiplying the corresponding vertex with $W_{L}^{ \pm}$by a factor of $-\tan ^{2} \theta_{W}$ for each $Z$. In particular, these vertices are not enhanced by $g_{R} / g_{L}$. There are no "mixed" vertices with one $W_{L}$ and one $W_{R}$. For vertices involving a $Z_{R}$, the $Z_{R} W_{R}^{+} W_{R}^{-}$vertex is given by $\tan \theta_{W} \cot \theta_{R} / \cos \theta_{W}$ times the corresponding vertex for $Z W_{L}^{+} W_{L}^{-}$and is, therefore, enhanced with respect to the latter by a factor of $\cot \theta_{R}$. Finally the $Z Z_{R} W_{R}^{+} W_{R}^{-}$vertex can be obtained by multiplying the $Z Z W_{L}^{+} W_{L}^{-}$vertex by $-\tan ^{3} \theta_{W} \cot \theta_{R} / \cos \theta_{W}$.
The Feynman rules for couplings of gauge bosons to fermions are already given in Eqs. (8) and (9). The Feynman rules involving the scalars which couple to top and bottom proportionally to the top-quark mass can be extracted from the Yukawa Lagrangian:

$$
\begin{aligned}
\mathcal{L}_{Y}= & -m_{t}\left[\bar{t} t+\frac{1}{\sqrt{2} v}\left(\bar{t} t h_{1}+\bar{b} b h_{2}\right)-\frac{i}{\sqrt{2} v}\left(\bar{t} \gamma_{5} t \phi_{1}^{0}\right.\right. \\
& \left.+\bar{b} \gamma_{5} b \phi_{2}^{0}\right)+\frac{1}{v}\left(\bar{b}_{L} t_{R} \phi_{2}^{-}+\bar{b}_{R} t_{L} \phi_{1}^{-}+\bar{t}_{L} b_{R} \phi_{1}^{+}\right. \\
& \left.\left.+\bar{t}_{R} b_{L} \phi_{2}^{+}\right)\right]
\end{aligned}
$$

In terms of the physical scalars defined as in Eq. (A3) the couplings we need become

$$
\begin{aligned}
\mathcal{L}_{Y}= & -\frac{m_{t}}{v}\left[\cos \theta_{R}\left(\bar{b} P_{R} t H_{1}^{-}+\bar{t} P_{L} b H_{1}^{+}\right)\right. \\
& +\frac{v_{R}}{\sqrt{v_{R}^{2}+v^{2}}}\left(\bar{b} P_{L} t H_{2}^{-}+\bar{t} P_{R} b H_{2}^{+}\right)+\frac{1}{\sqrt{2}} \bar{b}\left(h_{2}\right. \\
& \left.\left.-i H_{1}^{0} \gamma_{5}\right) b\right] .
\end{aligned}
$$

The vertices of the form $\mathrm{ZHH}$ are obtained from the Lagrangian

$$
\begin{aligned}
\mathcal{L}= & \frac{i g_{L}}{2 \cos \theta_{W}}\left(2 \sin ^{2} \theta_{W}-\frac{v_{R}^{2}}{v^{2}+v_{R}^{2}}\right) Z^{\mu}\left(H_{2}^{-} \partial_{\mu} H_{2}^{+}\right. \\
& \left.-H_{2}^{+} \partial_{\mu} H_{2}^{-}\right) \frac{-i g_{L}}{2 \cos \theta_{W}} \cos 2 \theta_{W} Z^{\mu}\left(H_{1}^{-} \partial_{\mu} H_{1}^{+}-H_{1}^{+} \partial_{\mu} H_{1}^{-}\right) \\
& +\frac{g_{L}}{2 \cos \theta_{W}} Z^{\mu}\left(h_{2} \partial_{\mu} H_{1}^{0}-H_{1}^{0} \partial_{\mu} h_{2}\right) .
\end{aligned}
$$

Finally, the vertices of the form $Z W H$ can be read from the Lagrangian

$$
\mathcal{L}=-\frac{g_{R}^{2}}{\sqrt{2}} \frac{\sin \theta_{R}}{\sin \theta_{W}} \frac{v v_{R}}{\sqrt{v^{2}+v_{R}^{2}}} Z^{\mu} W_{R \mu}^{ \pm} H_{2}^{\mp} .
$$

\section{Loops involving gauge bosons} 1.

Here we present results for the individual diagrams in Fig.

\section{Figure 1(a)}

To check our results we first evaluate this diagram for the case of the standard model in unitary gauge. In this case the internal wavy lines are $W^{+}$and $W^{-}$and one finds 


$$
\begin{aligned}
\Gamma_{\mu}= & -i \frac{g}{2 \cos \theta_{W}} \gamma_{\mu} \frac{g^{2} \cos ^{2} \theta_{W}}{16 \pi^{2}}\left[\frac { 1 } { \epsilon } \left(\frac{-M_{Z}^{4}}{12 M_{W}^{4}}-\frac{4 M_{Z}^{2}}{3 M_{W}^{2}}+\frac{M_{t}^{2} M_{Z}^{2}}{4 M_{W}^{4}}\right.\right. \\
& \left.\left.+\frac{3 M_{t}^{2}}{2 M_{W}^{2}}\right)+f a_{L}\right] P_{L} .
\end{aligned}
$$

Notice that the first two divergent terms in Eq. (A8) that are not proportional to $M_{t}^{2}$ are not included in the finite quantity $\delta g_{L b}$ of Eq. (28). These divergent terms cancel against other contributions from the renormalization of $G_{F}, M_{Z}$ and $\alpha$ when one calculates observables such as the partial $Z$ width [24]. It is possible to obtain a simple expression for the finite part $f a_{L}$ in the limit $M_{W}^{2} / M_{t}^{2} \ll 1$. It is given by

$$
\begin{aligned}
f a_{L} \approx & -\frac{M_{t}^{2}}{8 M_{W}^{2}}\left\{\frac{1}{\cos ^{2} \theta_{W}}\left[2 \log \left(\frac{M_{t}^{2}}{M_{Z}^{2}}\right)-3\right]\right. \\
& \left.+12 \log \left(\frac{M_{t}^{2}}{M_{Z}^{2}}\right)-10\right\} .
\end{aligned}
$$

For our model, the terms that are enhanced by $\cot ^{2} \theta_{R}$ are obtained when the internal wavy lines are $W_{R}^{+}$and $W_{R}^{-}$, resulting in

$$
\begin{aligned}
\Gamma_{\mu}= & -i \frac{g}{2 \cos \theta_{W}} \gamma_{\mu} \frac{-g_{R}^{2} \sin ^{2} \theta_{W}}{16 \pi^{2}}\left[\frac { 1 } { \epsilon } \left(\frac{-M_{Z}^{4}}{12 M_{W_{R}}^{4}}-\frac{4 M_{Z}^{2}}{3 M_{W_{R}}^{2}}\right.\right. \\
& \left.\left.+\frac{M_{t}^{2} M_{Z}^{2}}{4 M_{W_{R}}^{4}}+\frac{3 M_{t}^{2}}{2 M_{W_{R}}^{2}}\right]+f a_{R}\right) P_{R} .
\end{aligned}
$$

The first two divergent terms in this expression, the ones not proportional to $M_{t}^{2}$, do not contribute to the vertex correction $\delta g_{R b}$ that we are computing because they are not enhanced by $\cot ^{2} \theta_{R}$. Although they appear to be proportional to $g_{R}^{2}$, they really are not when one considers the relations from Eq. (18). The finite part can be calculated numerically (we only present these results for the sum of all diagrams). For example, for $M_{W_{R}}=500 \mathrm{GeV}$, we find $f a_{R}=1.32$ with a renormalization scale $\mu=M_{Z}$.

It is possible to present approximate analytical results by taking $M_{Z}=0$ in the integrals. Doing so and expanding the resulting expression in powers of $M_{t}^{2} / M_{W_{R}}^{2}$ we find

$$
\begin{aligned}
-i & \frac{g_{L}}{2 \cos \theta_{W}} \frac{g_{R}^{2}}{16 \pi^{2}}\left(-\sin ^{2} \theta_{W}\right)\left[\frac{3}{2} \frac{M_{t}^{2}}{M_{W_{R}}^{2}} \frac{1}{\epsilon}+\frac{3}{2}+\frac{M_{t}^{2}}{M_{W_{R}}^{2}}\left\{\frac{11}{4}\right.\right. \\
& \left.\left.-\frac{3}{2} \log \left(\frac{M_{W_{R}}^{2}}{\mu^{2}}\right)\right\}\right] .
\end{aligned}
$$

Figure 1(b)

Once again we first evaluate Fig. 1 for the standard model in unitary gauge. In that case the internal wavy line is a charged $W$ and the intermediate state quarks are top. The result can be written in the limit $M_{W}^{2} / M_{t}^{2} \ll 1$ as

$$
\begin{aligned}
\Gamma_{\mu}= & -i \frac{g}{2 \cos \theta_{W}} \gamma_{\mu} \frac{g^{2}\left|V_{t b}\right|^{2}}{16 \pi^{2}} \frac{M_{t}^{2}}{M_{W}^{2}}\left\{\left[-\frac{1}{\epsilon} \log \left(\frac{M_{t}^{2}}{M_{Z}^{2}}\right)-\frac{1}{2}\right] g_{L t}\right. \\
& \left.+\left[\frac{1}{4 \epsilon}-\frac{1}{4} \log \left(\frac{M_{t}^{2}}{M_{Z}^{2}}\right)-\frac{1}{8}\right] g_{R t}\right\} P_{L} .
\end{aligned}
$$

For our model the only terms enhanced by $\cot ^{2} \theta_{R}$ are obtained with the intermediate wavy line representing a $W_{R}^{ \pm}$ and the intermediate state quark being top. As before, we present an approximate analytical result obtained by setting $M_{Z}=0$ in the loop integrals and expanding the resulting expression in powers of $M_{t}^{2} / M_{W_{R}}^{2}$. This yields

$$
\begin{aligned}
-i & \frac{g_{L}}{2 \cos \theta_{W}} \frac{g_{R}^{2}}{16 \pi^{2}}\left(\frac{1}{\epsilon} \frac{M_{t}^{2}}{M_{W_{R}}^{2}}\left(\frac{g_{L t}}{4}-g_{R t}\right)-\frac{3}{4} g_{R t}\right. \\
& +\frac{M_{t}^{2}}{M_{W_{R}}^{2}}\left\{\left[\log \left(\frac{M_{t}^{2}}{\mu^{2}}\right)-\frac{1}{2}\right] g_{R t}-\frac{1}{4}\left[\log \left(\frac{M_{t}^{2}}{\mu^{2}}\right)\right.\right. \\
& \left.\left.\left.+3 \log \left(\frac{M_{t}^{2}}{M_{W_{R}}^{2}}\right)+\frac{7}{2}\right] g_{L t}\right\}\right) .
\end{aligned}
$$

We only consider the case with $M_{Z} \neq 0$ numerically and include it in the sum of all diagrams in Fig. 4.

\section{Figure 1(c)}

Finally we evaluate the wave-function renormalization diagrams of Fig. 1(c). Once again we begin by considering the standard model in unitary gauge. For this diagram we can present an exact analytical result

$$
\begin{aligned}
1-Z_{b}^{-1}= & \frac{g^{2}}{16 \pi^{2}} P_{L}\left[\frac{3 M_{t}^{2}}{4 M_{W}^{2}} \frac{1}{\epsilon}-\frac{3 M_{t}^{2}}{4 M_{W}^{2}} \log \left(\frac{M_{t}^{2}}{\mu^{2}}\right)\right. \\
& -\frac{3 M_{t}^{2} M_{W}^{2}}{4\left(M_{t}^{2}-M_{W}^{2}\right)^{2}} \log \left(\frac{M_{W}^{2}}{M_{t}^{2}}\right) \\
& \left.-\frac{6 M_{W}^{4}+5 M_{t}^{2} M_{W}^{2}-5 M_{t}^{4}}{8 M_{W}^{2}\left(M_{t}^{2}-M_{W}^{2}\right)}\right] .
\end{aligned}
$$

Similarly, for the case of our model, the terms that are enhanced by $\cot ^{2} \theta_{R}$ are obtained from the exchange of charged $W_{R}^{ \pm}$gauge bosons and we find

$$
\begin{aligned}
1-Z_{b}^{-1}= & \frac{g_{R}^{2}}{16 \pi^{2}} P_{R}\left[\frac{3 M_{t}^{2}}{4 M_{W}^{2}} \frac{1}{\epsilon}-\frac{3 M_{t}^{2}}{4 M_{W}^{2}} \log \left(\frac{M_{t}^{2}}{\mu^{2}}\right)\right. \\
& -\frac{3 M_{t}^{2} M_{W}^{2}}{4\left(M_{t}^{2}-M_{W}^{2}\right)^{2}} \log \left(\frac{M_{W}^{2}}{M_{t}^{2}}\right) \\
& \left.-\frac{6 M_{W}^{4}+5 M_{t}^{2} M_{W}^{2}-5 M_{t}^{4}}{8 M_{W}^{2}\left(M_{t}^{2}-M_{W}^{2}\right)}\right] .
\end{aligned}
$$

As a check of our calculation we have evaluated the corresponding expressions for the standard model in unitary 
gauge. From these we can obtain, by adding the three contributions, the vertex correction terms $\delta g_{L b}$ proportional to $M_{t}^{2}$. This result, Eq. (28), is finite and in agreement with the known result [18].

\section{Loops with scalar mesons}

We now turn our attention to the diagrams in Fig. 2. As described in the main text we use the basis of Eq. (A3) assuming all scalars to be degenerate and to have a large mass.

\section{Figure 2(a)}

This type of diagram involves one gauge boson and one physical charged scalar in the loop. There is only a contribution to the right-handed coupling involving the $W_{R}^{ \pm} H_{2}^{\mp}$ intermediate state. Our result in the limit where the Higgs boson masses are much larger than other masses is

$$
-i \frac{g_{L}}{2 \cos \theta_{W}} \frac{1}{16 \pi^{2}}\left(\frac{m_{t}}{v}\right)^{2}\left[\frac{1}{\epsilon}+\frac{3}{2}-\log \left(\frac{M_{H}^{2}}{\mu^{2}}\right)\right] x_{v}\left(1-x_{v}\right) P_{R} .
$$

\section{Figure 2(b)}

This type of diagram involves two scalars in the intermediate state. The left-handed coupling receives contributions from charged $H_{1}$ scalars as well as from a diagram with one neutral $H_{1}^{0}$ pseudo-scalar and one $h_{2}$ neutral scalar. The right-handed coupling receives contributions from the same diagram with neutral scalars as well as from the diagram with charged $\mathrm{H}_{2}$ scalars,

$$
\begin{aligned}
-i & \frac{g_{L}}{2 \cos \theta_{W}} \frac{1}{16 \pi^{2}}\left(\frac{m_{t}}{v}\right)^{2}\left[\frac{1}{\epsilon}+\frac{1}{2}-\log \left(\frac{M_{H}^{2}}{\mu^{2}}\right)\right] \\
& \times\left[\left(\sin ^{2} \theta_{W}+\cos ^{2} \theta_{W} x_{v}-\frac{1}{2} x_{v}^{2}\right) P_{R}\right. \\
& \left.+\left(-\frac{1}{2}-\frac{\cos ^{2} \theta_{R}}{2} \cos 2 \theta_{W}\right) P_{L}\right] .
\end{aligned}
$$

Figure 2(c)

This type of diagram involves the exchange of a charged $H_{1}$ or of a neutral $H_{1}^{0}$ or $H_{2}$ scalars for the left-handed coupling as well as exchanges of a charged $\mathrm{H}_{2}$ scalar or a neutral $A_{1}^{0}$ or $H_{2}$ scalars for the right-handed coupling,

$$
\begin{aligned}
-i & \frac{g_{L}}{2 \cos \theta_{W}} \frac{1}{16 \pi^{2}}\left(\frac{m_{t}}{v}\right)^{2}\left[\frac{1}{\epsilon}+\frac{1}{2}-\log \left(\frac{M_{H}^{2}}{\mu^{2}}\right)\right] \\
& \times\left\{\left[-\frac{1}{3} \sin ^{2} \theta_{W}+\left(-\frac{1}{2}+\frac{2}{3} \sin ^{2} \theta_{W}\right) x_{v}\right] P_{R}\right. \\
& \left.+\left(\frac{1}{3} \sin ^{2} \theta_{W}\left(1-2 \cos ^{2} \theta_{R}\right)\right) P_{L}\right\} .
\end{aligned}
$$

\section{Figure 2(d)}

This diagram represents $b$ wave-function renormalization through scalar loops. Once again, for the left-handed coupling one obtains contributions from exchanging a charged $H_{1}$ and neutral $H_{1}^{0}$ and $H_{2}$, whereas for the right-handed coupling the contributions arise through exchange of charged $\mathrm{H}_{2}$ and neutral $\mathrm{H}_{1}^{0}$ and $\mathrm{H}_{2}$ scalars,

$$
\begin{gathered}
-i \frac{g_{L}}{2 \cos \theta_{W}} \frac{1}{16 \pi^{2}}\left(\frac{m_{t}}{v}\right)^{2}\left[\frac{1}{\epsilon}+\frac{1}{2}-\log \left(\frac{M_{H}^{2}}{\mu^{2}}\right)\right] \\
\times\left[\left(-\frac{2}{3} \sin ^{2} \theta_{W}+\frac{1}{3} \sin ^{2} \theta_{W} x_{v}\right) P_{R}\right. \\
\left.+\left(1+\cos ^{2} \theta_{R}\right)\left(\frac{1}{2}-\frac{1}{3} \sin ^{2} \theta_{W}\right) P_{L}\right] .
\end{gathered}
$$

[1] ALEPH Collaboration, D. Abbaneo et al., hep-ex/0112021.

[2] Particle Data Group, K. Hagiwara et al., Phys. Rev. D 66, 010001 (2002).

[3] M.S. Chanowitz, Phys. Rev. Lett. 87, 231802 (2001).

[4] M.S. Chanowitz, Phys. Rev. D 66, 073002 (2002).

[5] G. Altarelli, F. Caravaglios, G.F. Giudice, P. Gambino, and G. Ridolfi, J. High Energy Phys. 06, 018 (2001).

[6] P. Bamert, C.P. Burgess, J.M. Cline, D. London, and E. Nardi, Phys. Rev. D 54, 4275 (1996).

[7] C.H. Chang, D. Chang, and W.Y. Keung, Phys. Rev. D 54, 7051 (1996).

[8] D. Chang and E. Ma, Phys. Rev. D 58, 097301 (1998).

[9] D. Choudhury, T.M. Tait, and C.E. Wagner, Phys. Rev. D 65, 053002 (2002).

[10] G. Burdman and D. Kominis, Phys. Lett. B 403, 101 (1997).
[11] C.X. Yue, Y.P. Kuang, X.L. Wang, and W.B. Li, Phys. Rev. D 62, 055005 (2000).

[12] G. Triantaphyllou, J. Phys. G 26, 99 (2000).

[13] D.J. Muller and S. Nandi, hep-ph/9610404.

[14] E. Malkawi and C.P. Yuan, Phys. Rev. D 61, 015007 (2000).

[15] E. Malkawi, T. Tait, and C.P. Yuan, Phys. Lett. B 385, 304 (1996).

[16] K.R. Lynch, E.H. Simmons, M. Narain, and S. Mrenna, Phys. Rev. D 63, 035006 (2001).

[17] X.G. He and G. Valencia, Phys. Rev. D 66, 013004 (2002); 66, 079901(E) (2002).

[18] Proceedings of the Workshop on Z Physics at LEP, Geneva, Switzerland, 1989, edited by G. Altarelli, R. Kleiss, and C. Verzegnassi (LEP, Geneva, 1989), Vol. 1.

[19] R.N. Mohapatra and J.C. Pati, Phys. Rev. D 11, 566 (1975); 11, 
2558 (1975); R.N. Mohapatra and G. Senjanovic, ibid. 12, 1502 (1975).

[20] K.S. Babu, X.G. He, and E. Ma, Phys. Rev. D 36, 878 (1987).

[21] Notice that there is a factor of 2 difference between the definitions of $\delta g_{R}$ here and in Ref. [17].
[22] A. Pukhov et al., hep-ph/9908288.

[23] Recall that the dependence of this amplitude on $\cot \theta_{R}$ is only through the width of the $Z^{\prime}$.

[24] S. Dawson and G. Valencia, Nucl. Phys. B439, 3 (1995). 